\title{
The Effects of Urbanization Process on Urban-Rural Income Gap based on the Feasible Generalized Least Squares
}

\author{
YANG Jun \\ School of Economics and Trade, Hunan University, Changsha 410079 \\ E-mail:junjun521@126.com
}

\begin{abstract}
This paper uses the method of empirical data to draw a trend analysis on the current situation and investigate the effect of urbanization on the income gap between urban and rural areas based on the data in 29 provinces in China from 1995 to 2010 and feasible generalized least squares method. Current situation analysis shows that urbanization is the important factors that affect the income gap between urban and rural areas. In the different stages of economic and social development, urbanization has different effect on the income gap between urban and rural areas. Empirical estimates show that urbanization significantly promoted China's income gap between urban and rural areas. For east, middle and west regions, the influence of urbanization on the local income gap also exists significant differences. Results show that different areas adjust measures to local conditions, and push the rural economic and social development through the urbanization is becoming an important approach to narrowing the income gap between urban and rural areas of China.
\end{abstract}

Keywords: urbanization, Feasible generalized least squares method, current situation, empirical analysis

\section{Introduction}

In recent years, China's economy has obtained the development which progresses by leaps and bounds, but at the same time, China's income gap between urban and rural areas has not improved, but rather in a rising trend after a brief decline [1]. Urbanization, on the other hand, as the important drivers of economic growth and industrial structure upgrade, will plays a more important role in China's economic and social development. Therefore, on the basis of combing the existing literature, combined with the status quo of the developing trend of Chinese urbanization, urban and rural income gap, this paper uses the empirical data of 29 provinces and cities in China to examine the national and eastern, central and western region urbanization impact on the income gap between urban and rural areas(1).

In terms of the relationship between urbanization and urban-rural income gap, the main existing literature is divided into three categories: one is that the urbanization can narrow the income gap between urban and rural areas, such as, the development economist Lewis theoretically expounds the urbanization is helpful to narrow the income gap between urban and rural areas [2]. Some domestic scholars, such as, Lu Ming and Chen Zhao came to a conclusion that there is an obvious relation between urbanization and urban-rural income gap urban, and urban bias of economic policy through the empirical analysis[3]. Xu Xiuchuan and Wang Zhao's study shows that urbanization is in a virtuous cycle with the narrowing of urban-rural income gap and non-agricultural labor force transfer [4]. The second category is that urbanization (or urbanization) enlarged the income gap between urban and rural areas, such as Lai Xiaodong thinks that according to measurement analysis, urbanization is related to income gap in a certain degree, and the widening of 
income gap moves together with the process of urbanization [5]. Cheng Kaiming and Li Jinchang argue that the urbanization and urban bias is the expanding cause of the income gap between urban and rural areas [6]. Chen Xun and Tong Jianhua argues that labor productivity difference between urban and rural areas, industrialization, unemployment and other factors caused the gap between urban and rural areas [7]. The third one is that urbanization (or urbanization) influences the income gap between urban and rural areas significant differently in different regions and economic developing stage, such as, Lin Yifu and Liu Mingxing use the provincial panel data from 1981 to 1997 to explore the factors affecting the income gap between urban and rural areas from the perspective of economic development strategy. They found that the influence of urbanization on the urban-rural income gap of residents is not sure [8]. Cao Yu's study shows that because most parts of China adopted a policy of urban and rural division of household register, it makes urbanization have both positive and negative influence on the urban-rural income gap [9].

In terms of the urbanization's influence on urban-rural income gap, the existing research is productive, but there are certain defects. First of all, in the aspect of the object of study, most of the research is based on the national level data. The analysis based on the research of regional level is relatively rare, based on the research of provincial area is much less. Secondly, on the research idea, although the existing research takes urbanization as an important factors to analyze the income gap between urban and rural areas, but most of the research did not consider the urbanization's dynamic effect on the income gap between urban and rural areas, the regional difference of urbanization on the income gap between urban and rural areas is seldom involved. Finally, on the research methods, the existing research pays less attention to the panel data of different variance and sequence relating problems, and the associated problems will have a significant impact on the empirical results' reliability. Therefore, this paper calculates scientifically 29 provincial areas' urban-rural income gap level in China in 1995-2010, which makes it possible to study the impact of urbanization on urban-rural income gap from the regional point of view. Then, by adding quadratic term of urbanization, this paper examines the urbanization's dynamic effect on the urban-rural income gap in China. And based on the related provinces, according to economic and geographic factors in eastern, central and western region classification, this paper has carried on a comparative analysis on the differences of three kinds of influence of regional urbanization on urban-rural income gap. Finally, on the basis of general panel data estimation, with the feasible generalized least squares method (FGLS) estimates, some existing problems can be well solved in the present study.

\section{An Empirical Analysis of Urban-Rural Income Gap Based On The Feasible Generalized Least Squares Urbanization}

\subsection{Model Construction}

In order to investigate the influence of urbanization on the income gap between urban and rural areas, on the reference to numerous empirical results of domestic research on urban-rural income gap, such as Xu Xiuchuan, Wang Zhao (2007)and Cao Yu(2010) etc., the present research constructs a model of measurement in this paper, the basic framework is as follows:

$$
r=a^{*}(u r b)^{b} *(p g d p)^{c} *(e x)^{d} *(g o v)^{e} * h^{f}
$$

Among them, the $r$ said the income gap between urban and rural areas, i.e. the ratio of urban per capita disposable income and the per capita net income of rural residents. Urb stands for the urbanization rate, $p g d p$ said wealth per capita, $e x$ is economic openness, gov ownership structure, and $h$ is human capital. 
In order to directly obtain the coefficient of the influence of factors on the income gap between urban and rural areas, at the same time to eliminate heteroscedasticity better, formula (2) can be got from formula (1) with the exponential on both sides:

$$
\ln r=\ln a+b \ln (u r b)+c \ln (p g d p)+d \ln (e x)+e(g o v)+f h
$$

Among them, $b 、 c 、 d 、 e 、 f$ directly reflects influence factors on the income gap between urban and rural areas, such as the urbanization, economic growth, foreign economic openness, ownership structure, human capital etc., and $\ln a$ is constant.

According to the Kuznets curve theory of income distribution, income gap will appear the inverted u-shaped curve with economic growth.

In order to study the urbanization and urban-rural income gap may have this kind of nonlinear relations, this article introduced income per capita ( $p g d p)$ 's second order items to the explanatory variables

$$
\begin{aligned}
\ln r_{i, t}= & c+\beta_{1} \ln (\text { urb })_{i, t}+\beta_{2} \ln (\text { pgap })_{i, t}+\beta_{3}[\ln (\text { pgdp })]_{i, t}^{2}+\beta_{4} \ln (\text { ex })_{i, t} \\
& +\beta_{5} \ln (\text { gov })_{i, t}+\beta_{6} \ln (h)_{i, t}+\mu_{i, t}
\end{aligned}
$$

Among them, $i$ said areas, $t$ said the year, $c$ as the constant term, $u_{i, t}$ as the random error term.

\subsection{Measuring Method, Data and Variables}

Due to the usage of panel data in empirical analysis, this paper use Hausman test values as a model to choose fixed effect (FE) and random effects (RE). Considering the income gap between urban and rural areas as well as the related data of provinces and cities, and there may be different variance problem, the present research estimates the model on the basis of ordinary least squares (OLS) and the cross - section weighted feasible generalized least squares method (FGLS). Meanwhile, considering the influence of regional differences, this article gives a regression estimate respectively to the eastern, central and western three areas urbanization influence factors at the same time of metrological analysis was carried out on the national level, so as to get a more scientific study on the effect of urbanization on China's urban-rural income difference, and other reasons for large differences in each region in terms of income disparity between urban and rural areas.

In this paper, the data mainly comes from China Statistical Yearbook in 1995-2011, China Rural Statistical Yearbook, China's Population Statistics Yearbook, China Education Statistical Yearbook and The New China 60 Years Statistical Data Collection. All involved in the form of value data, adopt corresponding price index of 1990 as the base for processing. Consider the availability and consistency of data, this article research object does not include Hong Kong, Macao and Taiwan regions, the Tibet autonomous region, and Chongqing.

The income gap between urban and rural areas $(r)$ in econometric model is represented by the ratio of urban residents per capita disposable income and per capita net income of rural residents, says the bigger the ratio, the greater the income gap between urban and rural areas. Otherwise, the smaller the ratio, the smaller the income gap between urban and rural areas. Urbanization rate (urban) is the ratio of urban population and regional population, the bigger the ratio, the higher the urbanization development level. Otherwise, the smaller the ratio, the lower the urbanization development level. The ratio of the GDP and population stands for Wealth per head $(p g d p)$ (ten thousand yuan as a unit).This index reflects the economic development. In terms of level of rural human capital (h), this 
paper adopts the practice of Zhang Xiaodi (2000), and calculats the culture condition of each province's rural household labor in the unit of year [13]. Ownership structure ( gov ) says the ratio of the number of staff in state-owned large and medium-sized enterprises and the total number of staff.

\subsection{Empirical Results and Analysis}

Table 1 is a statistical description of the variables for this paper. The table shows that the nation and its eastern, central and western regions have great differences in urban-rural income gap and corresponding influence factors. In terms of urban-rural income gap, the eastern is the smallest region in the country, nation and central are relatively close to each other, and western region has the largest income gap between urban and rural areas. In the aspect of urbanization rate, there is a decreasing trend in eastern, central and western. The urbanization rate in nation is lower than the east, but higher than the Midwest. Eastern wealth per person is higher than the Midwest. National wealth per capita is lower than the eastern part, but higher than the Midwest. Eastern economic openness is higher than the Midwest, national is lower than the eastern part of the country, but higher than the Midwest. Human capital has a comparative advantage relative to the eastern Midwest. The rate of State-owned large and medium-sized enterprises in central proportion is higher than the east and west, the national is lower than the west, but higher than the Middle East.

Table 1. Statistical Descriptions Of The Variables

\begin{tabular}{cccccccc}
\hline \multirow{2}{*}{ Region } & Variable & lnr & lnurb & $\begin{array}{c}\text { lnpgdp } \\
\text { RMB ten }\end{array}$ & $\begin{array}{c}\text { lnex } \\
\%\end{array}$ & lnh & lngov \\
& Unit & $\%$ & $\%$ & $\begin{array}{c}\text { year } \\
\text { thousand/person }\end{array}$ & $\%$ \\
\hline \multirow{5}{*}{ Nation } & Mean & 1.02 & 3.61 & -0.20 & 3.68 & -0.23 & 3.60 \\
& Standard Deviation & 0.22 & 0.41 & 0.54 & 0.39 & -0.27 & 0.68 \\
& Minimum & 0.47 & 2.60 & -1.3 & 2.74 & -1.05 & 1.20 \\
& Maximum & 1.56 & 4.49 & 1.16 & 4.49 & 0.27 & 4.87 \\
& Mean & 0.87 & 3.80 & 0.43 & 3.94 & 0.16 & 3.18 \\
& Standard Deviation & 0.14 & 0.46 & 0.32 & 0.80 & 0.21 & 0.65 \\
& Minimum & 0.47 & 2.84 & 0.01 & 2.11 & -0.73 & 1.44 \\
& Maximum & 1.15 & 4.49 & 1.16 & 5.32 & 0.19 & 4.54 \\
& Mean & 0.97 & 3.60 & -0.42 & 2.18 & -0.19 & 3.78 \\
& Standard Deviation & 0.14 & 0.29 & 0.23 & 0.29 & 0.24 & 0.62 \\
& Minimum & 0.55 & 2.84 & -0.83 & 2.84 & -0.66 & 1.20 \\
& Maximum & 1.19 & 4.01 & 0.14 & 4.02 & 0.22 & 4.87 \\
& Mean & 1.24 & 3.40 & -0.59 & 2.18 & -0.33 & 3.90 \\
& Standard Deviation & 0.16 & 0.34 & 0.33 & 0.42 & 0.32 & 0.52 \\
& Minimum & 0.76 & 2.60 & -1.30 & 1.26 & -1.05 & 1.89 \\
& Maximum & 1.56 & 4.01 & 0.54 & 3.60 & 0.27 & 4.50 \\
\hline
\end{tabular}

Table 2. Spearman Correlation Coefficients between Variables

\begin{tabular}{ccccccc}
\hline & $\ln r$ & $\operatorname{lnggdp}$ & $\operatorname{lnurb}$ & $\operatorname{lnex}$ & $\operatorname{lnh}$ & lngov \\
\hline $\ln r$ & 1.0000 & & & & & \\
$\ln p g d p$ & -0.5602 & 1.0000 & & & & \\
lnurb & -0.2998 & 0.4466 & 1.0000 & & & \\
lnex & -0.1963 & 0.4649 & 0.7910 & 1.0000 &
\end{tabular}




\begin{tabular}{ccccccc}
$\operatorname{lnh}$ & 0.0993 & 0.4616 & 0.4817 & 0.3889 & 1.0000 & \\
$\operatorname{lngov}$ & 0.1486 & -0.4997 & -0.2484 & -0.0927 & -0.3953 & 1.0000 \\
\hline
\end{tabular}

Table 2 shows spearman correlation coefficients between variables. It shows that the explanatory variables does not exist serious linear correlation.

Table 3 reports the analysis of regression and related inspection results. Equation 1 and 2 is the analysis of urban-rural income gap to the national level and of the influence factors of urbanization. Equation 3-4, 5-6, and7-8 are respectively analysis of urban-rural income gap and related factors of urbanization in the eastern, central and western regions. At the same time, in order to eliminate the influence of heteroscedastic and serial correlation better, equation 2, 4, 6, 8 use FGLS to robust estimation based on the fixed effect (or random effects) estimation. Hausman test results show that fixed effect estimation is used in the nation and the eastern region, while the central and western regions use the method of random effects.

In the national level, according to the results, urbanization and urban-rural income gap significantly positively related to each other. It illustrates the increase of urbanization will lead to income disparity between urban and rural areas to expand. The research results are close to Cheng kaiming and Li Jinchang [6], Chen Xun and Tong Jianhua [7], but opposite to the research results of Lu Ming and Chen Zhao [3], Xu Xiuchuan and Wang Zhao [4]. This may be the result of urbanization lead to the resources liquidity preference to city. Per capita income is significantly related to urban-rural income gap, its second order term is positively related to the urban-rural income gap as well. This shows that the Kuznets curve of urban-rural income gap may not exist in the process of changes in per capita wealth. This means that the initial per capita wealth increase may lead to the expansion of urban-rural income gap, and when the per capita wealth extents to a certain degree, the per capita growth in wealth will lead to widening income gap between urban and rural areas. Economic openness (ex) and the urban-rural income gap show the negative correlation. This shows that the promotion in economic openness can narrow the income gap between urban and rural areas to some extent. The human capital level significantly enlarges the urban-rural income gap. This is because the stock of human capital for the expansion of income gap effect is greater than the effect of equality. It presents residents income inequality situation increases with the human capital investment [14]. Proportion of state-owned large and medium-sized enterprises employees and urban-rural income gap show a significant negative correlation. It indicates that the state-owned large and medium-sized enterprises bring down the income gap between urban and rural areas, smaller even aggravate the influence of income gap between urban and rural areas.

Table 3. Results of Regression and Inspection

\begin{tabular}{ccccccccc}
\hline Region & \multicolumn{2}{c}{ Nation } & \multicolumn{2}{c}{ Eastern } & \multicolumn{2}{c}{ Central } & \multicolumn{2}{c}{ Western } \\
Equation & 1 & 2 & 3 & 4 & 5 & 6 & 7 & 8 \\
\hline \multirow{2}{*}{ lnurb } & $0.070^{* * * *}$ & $0.034^{*}$ & $0.076^{* * *}$ & $0.039^{* *}$ & $-0.094^{*}$ & -0.055 & 0.038 & 0.020 \\
& $(2.60)$ & $(1.72)$ & $(3.06)$ & $(1.98)$ & $(-1.68)$ & $(-1.17)$ & $(0.69)$ & $(0.37)$ \\
lnpgdp & $0.122^{* * *}$ & 0.028 & 0.057 & 0.068 & 0.171 & 0.117 & $0.149^{* *}$ & $0.136^{* *}$ \\
& $(3.85)$ & $(0.97)$ & $(0.479)$ & $(0.75)$ & $(1.55)$. & $(1.06)$ & $(2.71)$ & $\left.(2.13)^{* * *}\right)$ \\
& $0.148^{* * *}$ & $0.126^{* * *}$ & 0.961 & -0.038 & $0.477^{* * *}$ & $0.406^{* *}$ & $0.157^{* * *}$ & $0.169^{* * *}$ \\
$(\text { lnpgdp })^{2}$ & $(5.29)$ & $(4.45)$ & $(1.20)$ & $(-0.43)$ & $(2.93)$ & $(2.51)$ & $(0.008)$ & $(2.90)$ \\
& $-0.022^{*}$ & $-0.005^{*}$ & $0.110^{* * *}$ & $0.071^{* * *}$ & $0.139^{* * *}$ & $0.116^{* * *}$ & 0.009 & 0.004 \\
lnex & $(-1.73)$ & $(1.66)$ & $(6.09)$ & $(3.77)$ & $(4.64)$ & $(4.07)$ & $(0.34)$ & $(0.15)$ \\
& $0.243^{* * *}$ & $0.317^{* * *}$ & $-1.574^{* * *}$ & $-0.230^{* * *}$ & $0.352^{* * *}$ & $0.432^{* * *}$ & 0.074 & $0.257^{* * *}$ \\
lnh & $(8.22)^{* *}$ & $(10.45)$ & $(3.74)$ & $(-4.90)$ & $(5.09)$ & $(7.32)$ & $(0.95)$ & $(4.73)$ \\
lngov & $-0.005^{* *}$ & $-0.008^{* * *}$ & $-0.022^{* *}$ & $-0.032^{* *}$ & 0.011 & 0.004 & 0.003 & $0.040^{*}$
\end{tabular}




\begin{tabular}{ccccccccc} 
& $(-2.50)$ & $(-2.80)$ & $(-1.81)$ & $(-2.35)$ & $(0.62)$ & $(0.22)$ & $(1.07)$ & $(1.95)$ \\
$\mathrm{c}$ & $0.903^{* * *}$ & $0.973^{* * *}$ & 0.193 & $0.569^{* * *}$ & 0.294 & $0.823^{* *}$ & $1.02^{* * *}$ & $1.09^{* * *}$ \\
$\mathrm{R}^{2}$ & $(8.35)$ & $(8.32)$ & $(1.54)$ & $(4.26)$ & $(0.71)$ & $(2.35)$ & $(4.11)$ & $(4.39)$ \\
$\mathrm{F}(\mathrm{W})$ & 0.544 & 0.517 & 0.707 & 0.671 & 0.681 & 0.676 & 0.465 & 0.755 \\
& 85.42 & 393.46 & 64.02 & 113.82 & 40.63 & 237.62 & 20.86 & 122.63 \\
$\mathrm{H}$ & {$[0.000]$} & {$[0.000]$} & {$[0.000]$} & {$[0.000]$} & {$[0.000]$} & {$[0.000]$} & {$[0.000]$} & {$[0.000]$} \\
样本量 & 119.53 & 246.31 & 5.67 & 1.51 \\
& {$[0.000]$} & {$[0.000]$} & {$[0.461]$} & {$[0.959]$} \\
\hline
\end{tabular}

Note: () for the t statistics, $* * *, * * *$, respectively $10 \%, 5 \%, 1 \%$ significant level; [] to the corresponding $\mathrm{P}$ statistics; $\mathrm{H}$ is a Hausman test value and its probability.

Estimation in different regions show that urbanization, per capita income and other related factors have significant different influence on the regional urban-rural income gap. In the aspect of urbanization, the eastern region urbanization and urban-rural income gap is significant positive correlation. It demonstrates that the eastern region urbanization expanded the income gap between urban and rural areas. This could result from higher urbanization and economic and social development level in the eastern region. In the process of urbanization, some farmers with good education or business ability turned to citizens or urban resident population, which increased the income gap between urban and rural areas [3]. Urbanization in central China and the urban-rural income gap are of significant negative correlation, which indicates that the urbanization has smooth effect on the urban-rural income gap in the central region, but not very significant. This may be because the central rural economy foundation is weak, which has certain positive influence on increasing the income of the rural population urbanization [6]. Western region urbanization and urban-rural income gap is a significant positive correlation, which shows that urbanization has less effect on the urban-rural income gap. It may be because the limitation in west traffic, economic basis and other factors, and resources have certain city bias effect in the process of urbanization [7].

\section{An Analysis on the Present Trend of Urbanization and Urban-rural Income Gap Trend}

For a more intuitive presentation of China and its Eastern, central and western regions in urbanization and the changing trend of the urban-rural income gap, the author draws a Chinese urbanization and urban and rural income gap trend chart is as follows according to the statistical data in China Statistical Yearbook (1995-2010).

\subsection{The Changing Trend of Urbanization}

The Figure 1 shows that China's urbanization is in the world urbanization development trajectory. As Novartis has pointed out that the development of the urbanization process is periodic one, just like a flattening S type curve [10]. Some scholars divided urbanization rate into three stages [11], namely, preparing stage (urbanization level below 30\%), which in a slow speed of change; Developing stage (30\% 70\%), which speeds up in changing; Mature stage (over 70\%), which hastens to slow down the speed of change. Reference to the above classification, it can be seen from Figure that China's overall level of urbanization is between $30 \% \sim 70 \%$, which in the developing stage in the urbanization overall. China's eastern, central and western urbanization development level is not balanced: Central east is much higher than the national average, slightly lower than the national average. The west is far lower than the national average level, whose urbanization level in 2010 was worth only east around 2000. According to the data from the National Bureau of Statistics, at the end of 2011, China's urbanization rate reached $51.27 \%$. The eastern urbanization rate has exceeded 60\%, and the Midwest is still in $30 \%$ 
to $50 \%$ range, which shows that the Midwest is still in the high-speed economic development. What also can be seen from the Figure 1 is that the national urbanization was in a steady growth stage from 1995 to 1999, reached turning point around 2000, and grew faster in 2000-2010. Combined with the current development trend, China's urbanization rate will remain to accelerate in the future, and the economy development differences in eastern, central and western regions are difficult to narrow in the short term.

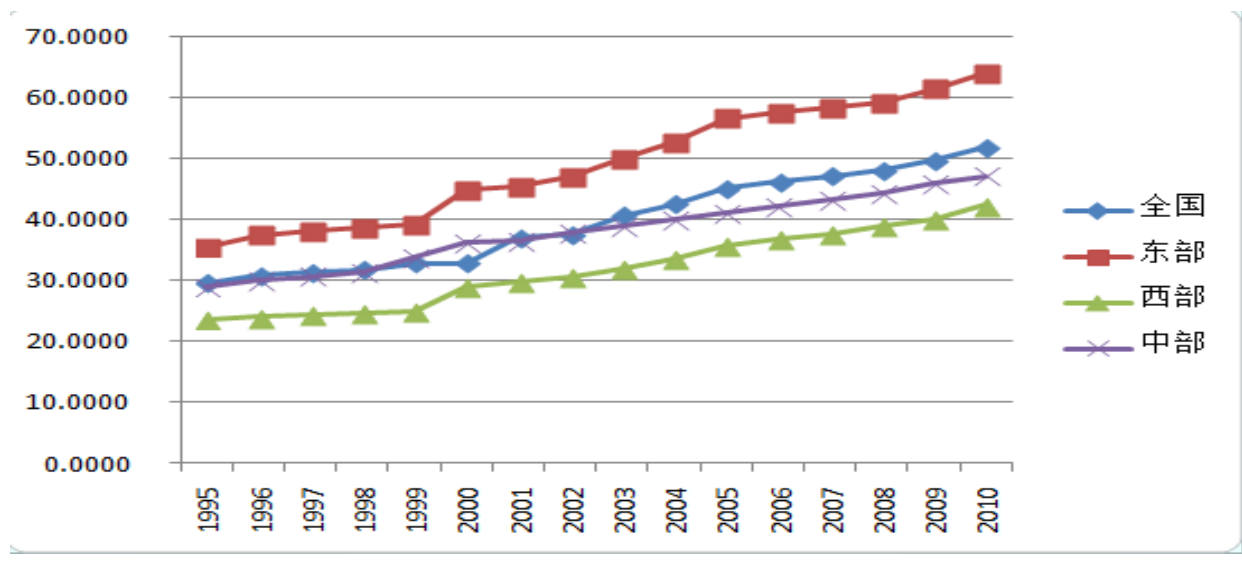

Figure1. Urbanization Rate in the Nation and Eastern, Central, Western
Regions

\subsection{The Income Gap between Urban and Rural Areas Changing Trend}

Since China's reform and opening up, the urban-rural income gap has been widening with China's rapid economic and social development. According to the China City Development Report released by the Urban Development and Environment Research Institute in Chinese Academy of Social Sciences, China's urban-rural income gap ratio at present is 3.23:1, which has become one of the countries with largest income gap between urban and rural areas in the world. Figure 2 shows changes of urban-rural income gap in the national and eastern, central and western regions in 1995-2010. The Figure 2 shows that China's overall and eastern, central and western urban-rural income gap is almost in the same changing trend, among which the western region enjoys the highest income gap between urban and rural areas, the central and eastern is lower than the national average. The income gap between urban and rural areas in China appeared inflection point around 1998, which in turn showed a trend of accelerating after 1998. This may be connected with the macroeconomic policy adjustment, the reform of state-owned enterprises salary and other factors. Around 2005, the income gap between urban and rural areas showed a trend of slow rise and fall. This may be related to China's vigorous pushing relevant supporting policies to the "Three Rural". 


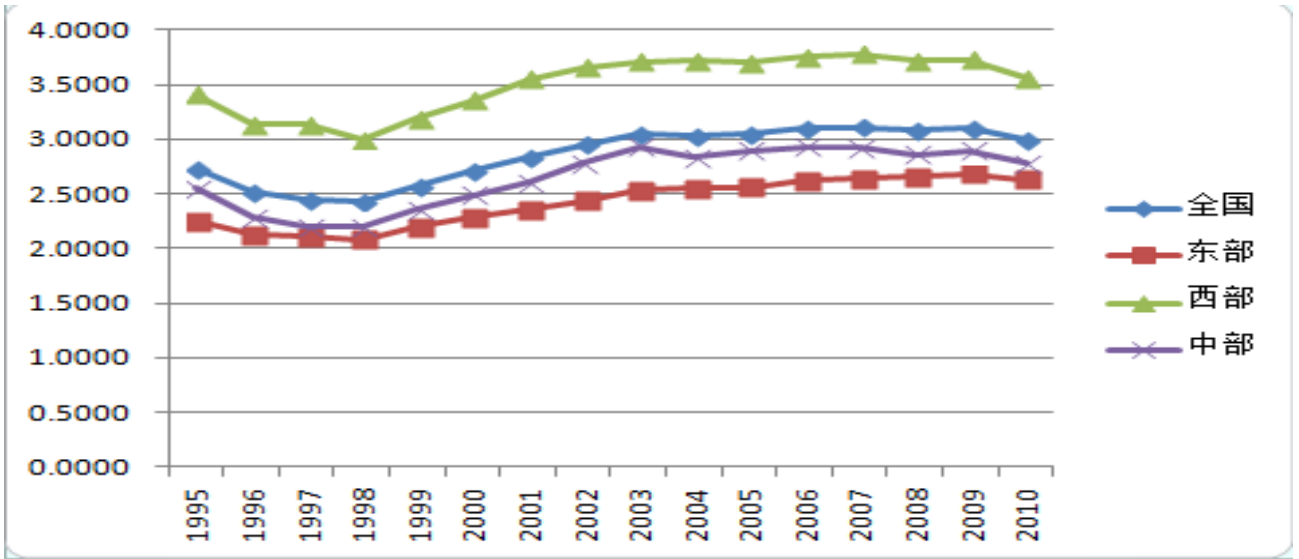

\section{Figure 2. Urban and Rural Income Ratio of National, Eastern, Central and Western Regions (1995-2010)}

Figure 1 and Figure 2 show that China's overall urbanization and the urban-rural income gap is in a trend of unstable change before 1999, and the growth of urbanization existed with the narrowing of urban-rural income gap; From 1999 to 2005, the growth of urbanization and the widening of urban-rural income gap coexisted; 2005 years later, urbanization was in promotion while the urban and rural income gap was narrowing. This shows that China's urbanization impact mechanism on the urban-rural income gap is more complex. Eastern and western urbanization and urban-rural income gap has been changing in the same direction which could widen the income gap between urban and rural areas. Urbanization and urban-rural income gap in central trend presents the changes in the opposite direction, the local urbanization may narrow the income gap between urban and rural areas. This means that the urbanization impact on China's income gap between urban and rural areas has significant regional differences, so it is quite necessary to study the issue from a regional level.

\section{Conclusion}

Based on the data in 29 provinces in China from 1995 to 2010 and feasible generalized least squares method, this paper uses the method of empirical data to draw a trend analysis on the current situation and investigate the effect of urbanization on the income gap between urban and rural areas. Research results show that urbanization, on the whole, expanded the urban-rural income gap, but the influence of urbanization on it has significant regional differences: east urbanization and urban-rural income gap is in significant positive correlation, while central and western urbanization and urban-rural income gap is in a not significant positive correlation. Economic growth on the whole enlarged the urban-rural income gap. Economic growth and the urban-rural income gap of eastern and central not very significant positive correlation, but the west is very significantly correlated. Economic openness and the urban-rural income gap is significantly negative correlation, open economy is beneficial to reduce the urban-rural income gap. Human capital and the urban-rural income gap is significantly related, and the human capital expands the income gap between urban and rural areas. The ratio of state-owned large and medium-sized enterprises and the urban-rural income gap are in negative correlation, among which the east is significantly negative correlation, and the central and western positive correlation is not significant.

\section{References}

[1] Y.-M. Chen, "How Much Does Country Matter? The Estimation of Variance in High-tech Industry Performance", International Regional Science Review, vol. 4, no. 31, (2008). 
[2] C.-T. Tan, "Development Economic", Taiyuan: Shanxi Economic press, (2001).

[3] M. Lu and Z. Chen, "Urbanization, Urban-Biased Economic Policies and Urban-Rural Inequality", Economic Research Journal, (2004).

[4] X.-C. Xu and Z. Wang, "Empirical Study on the Interactive Relationship among Urbanization, Industrialization and Urban-Rural Income Disparity in China", Issues in Agricultural Economy, (2008).

[5] L. Xiao-Dong, "A Research on the Relationship Between Regional Income Disparity and Urbanization", Journal of Southwest Jiao tong University (Social Sciences), (2005).

[6] K.-M. Cheng and J.-C. Li, "Analysis of Mechanism and Dynamic Econometric Relationships between Urban Bias, Urbanization and Urban-rural Inequality", The Journal of Quantitative \& Technical Economics, (2007).

[7] X. Chen and H.-J. Tong, "On Urbanization and Income Gap between Urban and Rural Areas", Productivity Research, (2007).

[8] Y.-F. Lin and M.-X. Liu, "Growth Convergence and Income Distribution in China", World Economy, (2003).

[9] Y. Chao, X.-H. Chen and Y.-R. Ma, "Urbanization, Urban-Rural Income Gap and Economic Growth”, Statistical Research, (2010).

[10] R. M. Northam, "Urban Geography”, New York: John Wiely\& Sons, (1979).

[11] N.-R. He and Y. Wu, "Theoretical Study of Chinese Contemporary Urbanization”, Shanghai: Shanghai People's press, (2007).

[12] G.-H. Huang, "Impact Factors Analysis about External Labor Inflow Based on the Yangtze River Delta", China Population Resources and Environment, (2010).

[13] B.-L. Jiao, "Influence of Human Capital Structure on the Income Gap of Residents", Chinese Journal of Population Science, (2011).

[14] X.-Y. Qian and X.-D. Zhang, "Rural Human Capital Accumulation and Income Characteristics", Chinese Rural Economy, (2000).

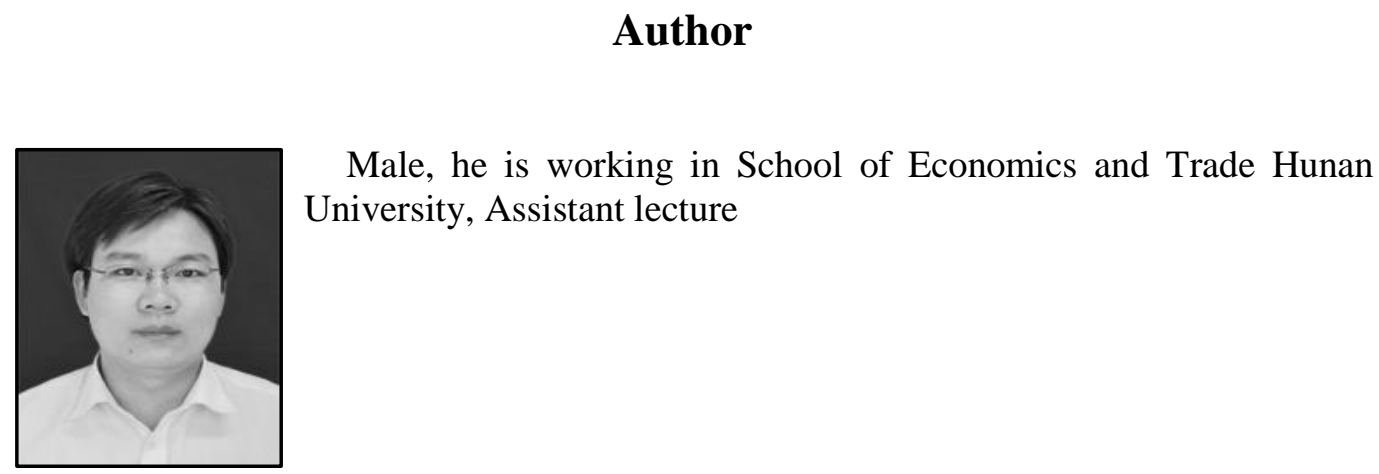


International Journal of $u-$ and e- Service, Science and Technology Vol.8, No. 10 (2015) 